\title{
Desarrollo empresarial e innovación en el sector de manufacturas plásticas zulianas*
}

\author{
Business development and innovation in the zulian plastic manufactures sector \\ Desenvolvimento empresarial e inovação no setor de manufaturas plásticas zulianas
}

DOI: https://doi.org/10.21803/pensam.v11i21-1.271

Mariby Boscán

https://orcid.org/0000-0002-4974-812X

Maryana Sandrea

\section{¿Cómo citar este artículo?}

Boscán, M. \& Sandrea, M. (2018). Desarrollo empresarial e innovación en el sector de manufacturas plásticas zulianas Pensamiento Americano, 11(21), 154-165.

\section{Resumen}

Las exigencias cambiantes del entorno y las transformaciones que viven las empresas requieren la definición de estrategias adecuadas, que permitan alcanzar ciertas condiciones de avanzadas, como la incorporación de innovaciones en el sistema productivo, desarrollar cambios tecnológicos, alta calificación del recurso humano, variables clave para impulsar y consolidar el desarrollo empresarial. En este sentido, el objetivo general de la presente investigación consiste en determinar el desarrollo empresarial y la innovación en el sector de manufactureras plásticas zulianas. El estudio planteado es descriptivodocumental y de campo, con un diseño transeccional, no experimental. Para la recolección de data se empleó la revisión de fuentes secundarias y entrevistas a algunos gerentes del sector. Entre los resultados destacan: El $66,7 \%$ de los empresarios estimulan la difusión de innovaciones y de conocimiento en $83,3 \%$, asimismo consideran el desarrollo urbano $(66,7 \%)$ y el entorno institucional $(79,9 \%)$ favorables para el desarrollo endógeno. La mayoría de las empresas cuentan con personal capacitado (86,7\%) y un nivel tecnológico apropiado (76,6\%), sin embargo, el 66,6\% no desarrollan cambios tecnológicos, ni propicia generación propia de tecnología (76,7\%). Finalmente, para impulsar el desarrollo del sector destacan estrategias gerenciales para el desarrollo tecno-endógeno, que impulsan la transformación y renovación del sistema productivo local.

Palabras Claves: Desarrollo empresarial, estrategias gerenciales, innovación, sector de manufacturas plásticas zuliano, sistema productivo.

\begin{abstract}
The changing demands of the environment and the changes experienced by companies require the definition of adequate strategies that allow certain advanced conditions to be met, such as the incorporation of innovations in the productive system, development of technological changes, high qualification of human resources, key variables to promote and consolidate business development. In this sense, the general objective of this research is to determine the business development and innovation in the sector of Zulian plastic manufacturers. The proposed study is descriptive-documentary and field, with a transectional design, not experimental. For the collection of data, the review of secondary sources and interviews with some managers of the sector was used. The results include: $66.7 \%$ of entrepreneurs encourage the dissemination of innovations and knowledge in $83.3 \%$, and also
\end{abstract}

*Artículo de investigación resultado del proyecto titulado: "gestión ambiental y reconversión productiva en empresas de manufacturas plásticas", n de oficio vac-condesch- 0460-15 y realizado desde el 01 de mayo del 2015 (en curso). 
consider urban development (66.7\%) and the institutional setting (79.9\%) favorable for endogenous development. Most of the companies have trained personnel (86.7\%) and an appropriate technological level (76.6\%); however, $66.6 \%$ do not develop technological changes or promote their own generation of technology (76.7\%). \%). Finally, in order to promote the development of the sector, management strategies for technoendogenous development stand out, which drive the transformation and renovation of the local productive system.

Key words: Business development, management strategies, innovation, Zuliano plastic manufacturing sector, production system.

\section{Resumo}

As exigências rotativas do entorno e as transformações que vivem as empresas requerem a definição de estratégias adequadas, que permitam alcançar certas condições como a incorporação de inovações no sistema produtivo, desenvolver mudanças tecnológicas, alta qualificação de recursos humanos, variáveis para impulsionar e consolidar o desenvolvimento empresarial. Neste sentido, o objetivo geral da presente pesquisa consiste em determinar o desenvolvimento empresarial e a inovação no setor de manufatureiras plásticas zulianas. O estudo é de tipo descritivo-documental e de campo, com um desenho transeccional, não experimental. Para coletar a informação, houve uma revisão de fontes secundárias e entrevistas a alguns gerentes do setor. Entre os resultados, se destacam: El 66,7\% dos empresários estimulam a difusão de inovações, e de conhecimento um 83,3\%. Mesmo assim, consideram o desenvolvimento urbano $(66,7 \%)$ e o entorno institucional $(79,9 \%)$ favoráveis para o desenvolvimento endógeno. A maioria das empresas contam com pessoal capacitado $(86,7 \%)$ e um nível tecnológico apropriado (76,7\%). Finalmente, para impulsar o desenvolvimento do setor, destacam-se estratégias gerenciais para o desenvolvimento tecno-endógeno, que impulsionam a transformação e renovação do sistema produtivo local.

Palavras-chave: Desenvolvimento empresarial, estratégias gerenciais, inovação, setor de manufaturas plásticas zuliano, sistema produtivo.

\section{Perfil}

Magíster en Gerencia de Empresas - Mención Gerencia Financiera. Economista. Profesora Titular e Investigadora de la Universidad del Zulia, adscrita al Instituto de Investigaciones de la Facultad de Ciencias Económicas y Sociales. Coordinadora del Departamento de Estudios de Microeconomía. Editora de la Revista de Ciencias Sociales (RCS). maribyboscán@yahoo.com, boscan_mariby@fces.luz.edu.ve

\section{Perfil}

Magíster en Gerencia de Empresas- Mención Gerencia Financiera. Economista. Profesora Titular e Investigadora de la Universidad del Zulia, adscrita al Instituto de Investigaciones de la Facultad de Ciencias Económicas y Sociales. Departamento de Estudios de Microeconomía.

Email: marysandrea@yahoo.com

\section{Mariby Boscán}

Ph.D en Ciencias Gerenciales.

\section{Maryana Sandrea}




\section{Introducción}

L os cambios que se han dado en el paradigma tecno-económico han Ilevado al desarrollo de un modelo de competitividad en el cual el papel de las empresas queda determinado principalmente por su estrategia de crecimiento. Es por ello por lo que, actualmente se reconoce que la competitividad de las empresas se relaciona con su tamaño, factor al cual se asocia la capacidad para obtener grandes volúmenes de producción, realizar cuantiosas inversiones en bienes de capital, emprender costosas estrategias de comercialización o reducir los costos de la mano de obra, así como la organización flexible de los factores productivos y la actividad.

En este sentido, en los últimos años, Venezuela ha experimentado profundos cambios económicos, acompañados de una progresiva desestabilización tanto social como política. Por lo cual, el país enfrenta tres grandes desafíos: continuar, así como profundizar la reestructuración del aparato productivo privado y público para hacerlo más competitivo internacionalmente, modernizar al sector público para fortalecer su rol como ente regulador y consolidar el proceso de descentralización.

Por otro lado, las exigencias cambiantes del entorno y las transformaciones que viven las empresas, requieren la definición de estrategias adecuadas, obtención y buena administración de recursos financieros, que les permitan alcanzar ciertas condiciones o factores de avanzadas, como la incorporación de innovaciones en el sistema productivo, alta calificación del recurso humano, variables clave para impulsar y consolidar el desarrollo, así como mayores niveles de investigación y utilización de tecnología de vanguardia, entre otros, que le ayuden a fortalecerse y desarrollarse exitosamente (Boscán, 2013).
Dentro de este contexto, es importante resaltar que la dinámica e intensidad de las reformas impuestas por el cambio estructural, requieren del Estado una mayor capacidad de acción y organización. Esa capacidad debe ser definida como una potencialidad para obtener resultados mediante nuevos tipos de organizaciones en donde es necesario incorporar la óptica de gerencia empresarial como principio de manejo. Al respecto, el gerente debe formular estrategias efectivas y oportunas, para alcanzar sus objetivos generales, que permitan mejorar la productividad, y alcanzar el crecimiento sostenido en su empresa.

Por todo lo antes expuesto, es indiscutible la necesidad e importancia que tiene para el desarrollo económico del país, así como para su estabilidad política y social, el fortalecimiento de sectores productivos zulianos, razón por la cual se hace necesario determinar el desarrollo empresarial y la innovación en el sector de manufactureras plásticas zulianas.

Es importante destacar algunos aspectos que caracterizan las empresas objeto de estudio, lo cual permite establecer un marco general respecto al desempeño de las mismas: El sector de manufacturas plásticas, según Sandrea y Boscán (2010), comprende el conjunto de empresas dedicadas a producir bienes a base de compuestos orgánico-químicos poliméricos, obtenidos por síntesis a partir de derivados del petróleo, gas natural y carbón o procedentes de sustancias de origen vegetal como la celulosa y el almidón.

En Venezuela la crisis desencadenada y sostenida desde hace algunos años ha afectado los sectores de la economía, envolviendo así al sector dedicado a las manufacturas de plásticos ya que en los últimos años se denoto cierta caída en las ventas de los mismos. Es por ello que las empresas del sector ac-

Pensamiento Americano Vol. 11 (22) · 2018 • Julio-Diciembre · Corporación Universitaria Americana • Barranquilla, Colombia • ISSN: $2027-2448$. 
tualmente utilizan distintos medios para posicionarse en el mercado tomando en cuenta los recursos con los que cuenta para lograr sus objetivos. Asimismo, cabe mencionar que en el estado Zulia este sector productivo presenta múltiples problemas, destacándose según Boscán (2013): dificultades en el acceso a las materias primas, mantenimiento de altos márgenes de capacidad ociosa, unido a la rápida obsolescencia tecnológica, lo cual ha implicado altos costos para los empresarios, ya que las maquinarias y equipos que se utilizan son importados, problemas de financiamiento, entre otros.

Según los resultados preliminares de un estudio de campo actualmente en ejecución en el sector zuliano de manufacturas plásticas (considerando hasta el momento 10 organizaciones), el 60\% de las empresas presentan obsolescencia técnica de más de diez años, lo que implica una tendencia hacia altos niveles de obsolescencia en el sector.

\section{Método}

En cuanto a la metodología utilizada, se diseñó un estudio descriptivo, documental, y de campo con un diseño transeccional, no experimental, utilizando para la recolección de la data la revisión de fuentes secundarias y algunos resultados preliminares producto de una investigación de campo aún en ejecución, la cual consiste en la realización de entrevistas personales a la gerencia de algunas empresas del sector. En la actualidad se ha recabado la información correspondiente a 10 empresas (que dieron información a través de una entrevista personal) de un total de 30 (que existían en 2013), por lo cual la información de fuente primaria para el año 2018 presentada corresponde a una parte del sector.

Al respecto, Hernández, Fernández y Baptista (2010), afirman que los estudios descriptivos sirven para el análisis real y el comportamiento del fenómeno y sus componentes de manera independiente o conjunta, es decir pretenden medir o recoger información, sobre los conceptos o variables a las que se refieren. Por su parte, Hurtado (2012), sostiene que en las investigaciones descriptivas el propósito es exponer el evento estudiado, haciendo una enumeración detallada de sus características, de modo tal que en los resultados se puedan obtener dos niveles de análisis, dependiendo del fenómeno y del propósito del investigador: un nivel más elemental, en el cual se logra una clasificación de la información en función de características comunes, y un nivel más sofisticado en el cual se pone en relación los elementos observados a fin de obtener una descripción más detallada, siendo esta última la que se pretende en esta investigación.

Asimismo, se propone un diseño no experimental, transeccional, lo cual implica que la variable se observa tal y como se da en su contexto natural, para luego analizarla, es decir, se describe y analiza su incidencia e interrelación en un momento dado. Los objetivos se alcanzaron a partir de la revisión de fuentes secundarias especialmente libros, artículos científicos, tesis de grado, documentos de internet, entre otros, así como entrevistas a algunos gerentes del sector.

Finalmente, cabe mencionar que parte de los datos referidos en esta investigación se derivan del proyecto de investigación "Gestión ambiental y reconversión productiva en empresas de manufacturas plásticas", registrado en el Consejo de Desarrollo Científico y Humanístico de la Universidad del Zulia, dentro del Instituto de Investigaciones de la Facultad de Ciencias Económicas y Sociales, concerniente a la línea de investigación fundamentos microeconómicos para la toma de decisiones, aun en desarrollo. 


\section{Desarrollo e innovación}

Frente a la globalización, han surgido como esperanza para los países pobres, ideas de desarrollo, específicamente endógeno. Se trata según Ochoa (2006), de impulsar cambios económicos y sociales desde adentro, partiendo del territorio, así como de las capacidades locales, valorando los recursos existentes, dinamizando tanto a los actores como a los factores productivos, según estrategias consensuadas de integración en proyectos concretos, que favorezcan la creación de riqueza, su distribución, además el crecimiento económico y la mejora de la calidad de vida de la población. Vázquez (2018), sostiene que el desarrollo endógeno puede entenderse como un proceso sostenible de crecimiento económico y cambio estructural, llevado a cabo por la comunidad local utilizando el potencial de desarrollo interno, que busca aumentar el empleo, reducir la pobreza, satisfacer las demandas básicas de los ciudadanos, en suma, mejorar el nivel de vida de la población.

Para este autor (Vázquez, 2007: 188), la endogeneidad se entiende como la capacidad de la comunidad local para adoptar su propia estrategia de desarrollo, llevando a cabo las acciones necesarias para alcanzar los objetivos trazados por la sociedad. "Cada territorio tiene un conjunto de recursos materiales, humanos, institucionales y culturales que constituyen su potencial de desarrollo".

Reforzando a su vez, estas definiciones Mas (2006) y Ochoa (2006), estudian el desarrollo endógeno, como una estrategia y un fin. Es decir, como un vehículo que permite ajustar algunas variables para atender las distorsiones que se generan en un determinado territorio por la acción de fuerzas externas o como una forma alterna para generar un proyecto de sociedad distinto en el cual el desarrollo es un fin en sí mismo.
Asimismo, sostiene Vázquez (2007) que la teoría del desarrollo endógeno se diferencia de los modelos de crecimiento endógeno, en que integra el crecimiento de la producción en la organización social e institucional del territorio, adopta una visión territorial, no funcional de los procesos de crecimiento, así como del cambio estructural, por último, en que entiende que tanto los mecanismos como las fuerzas del desarrollo actúan sinérgicamente, condicionando la dinámica económica. Tiene, por tanto, una visión más compleja del proceso de acumulación de capital, que le lleva a plantearse las políticas de desarrollo económico desde el territorio, dándole a la sociedad civil un papel protagonista en la definición y ejecución del futuro de la economía.v

Por su parte, la innovación es entendida como la concepción y establecimiento de cambios significativos en el producto, el proceso, el marketing o la organización de la empresa, con el propósito de mejorar sus resultados. Los cambios innovadores se realizan mediante la aplicación de nuevos conocimientos y tecnología que pueden ser desarrollados internamente, en colaboración externa o adquiridos mediante servicios de asesoramiento o por compra de tecnología (OCDE, 2005 citado por Velázquez, Coria y Vargas, 2018).

En ese sentido, la innovación representa la suma total de un proceso social conjunto (y no el resultado en sí mismo), en cuya culminación se utiliza o no una invención. Para Rodríguez (2009), innovar es convertir ideas en productos, procesos o servicios nuevos o mejorados que el mercado valora, se trata de un hecho que incrementa la capacidad de creación de riqueza de la empresa y tiene además fuertes implicaciones sociales.

Por lo antes expuesto y tal como lo señalan Boscán y Sandrea (2006), los procesos de desarrollo son consecuencia entre otros, de la introduc-

Pensamiento Americano Vol. 11 (22) • 2018 • Julio-Diciembre • Corporación Universitaria Americana • Barranquilla, Colombia • ISSN: $2027-2448$. 
ción de innovaciones y conocimientos (tecnología blanda y dura). De allí la importancia de la incorporación de innovaciones tecnológicas (avances en el conocimiento sobre técnicas o en equipos físicos de producción), que impulsen la transformación y renovación del sistema productivo local, así como la posibilidad de crear una capacidad tecnológica propia, que imprima dinamismo y sustentabilidad al desarrollo económico del país.

Dado que las empresas no compiten aisladamente, sino que lo hacen juntamente con el entorno productivo e institucional del que forman parte, los procesos de acumulación de capital y desarrollo están condicionados por un conjunto de factores clave que actúan sinérgicamente. Por lo tanto, es indispensable indagar sobre los elementos determinantes del desarrollo, presentes en las empresas del sector de manufacturas plásticas zuliano. Para lo cual, se consideró la perspectiva explicada por Vázquez (2007), quien sostiene que es necesario activar los factores determinantes del desarrollo, como son la creación y difusión de las innovaciones y el conocimiento en el sistema productivo, el desarrollo urbano del territorio y la densidad del tejido institucional (entorno institucional), entre otros.

\section{Difusión de las innovaciones}

Tal como se puede apreciar en la tabla 1, el 66,7\% de los gerentes de las empresas de manufacturas plásticas zulianas, siempre (36,7\%), casi siempre $(20 \%)$ o algunas veces $(10 \%)$ ha estimulado la difusión de las innovaciones dentro de su empresa, en el esfuerzo por aumentar la rentabilidad de sus inversiones y ampliar su presencia en los mercados, mientras que el 33,3\% nunca lo ha incitado. Este comportamiento se apega a los planteamientos de Freeman y Soete, (1997) citado por Vázquez (2007), quienes sostienen que el desarrollo económico y la eficiencia productiva dependen de la introducción y difusión de las innovaciones que impulsan la transformación y renovación del sistema productivo. En este sentido, actualmente las empresas dependen de lo que sus competidores hacen, de las relaciones que las empresas mantienen con el entorno y del carácter innovador del mismo.

Tabla 1:

Estímulo a la difusión de innovaciones

\begin{tabular}{|c|c|c|}
\hline & Frecuencia & Porcentaje \\
\hline Casi Nunca & 10 & 33,3 \\
\hline Algunas Veces & 3 & 10,0 \\
\hline Casi Siempre & 6 & 20,0 \\
\hline Siempre & 11 & 36,7 \\
\hline Total & 30 & 100,0 \\
\hline
\end{tabular}

Fuente: Boscán (2013).

\section{Difusión de conocimientos}

En cuanto a si el empresario propicia la participación de los empleados en cursos de actualización de conocimientos técnicos, se muestra en la gráfica 1 que el $83,3 \%$ de los gerentes de las empresas de manufacturas plásticas siempre (36,7\%), casi siempre (33,3\%) o algunas veces $(13,3 \%)$ ha motivado la participación de sus trabajadores en cursos de actualización de conocimientos técnicos, mientras que el 16,7\% de los empresarios entrevistados manifestó que casi nunca propicia tal participación de sus empleados.

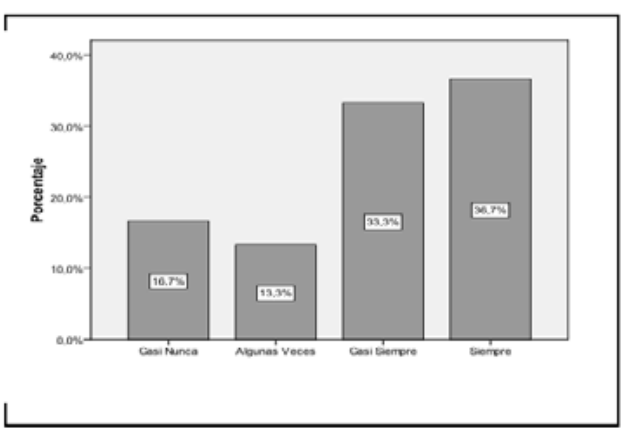

Gráfica 1: Participación de los empleados en cursos de actualización. Fuente: Boscán (2013). 
Este resultado muestra que la mayoría de los gerentes entrevistados están de acuerdo con los planteamientos de Petrizzo (2008), quien sostiene que la gestión del conocimiento, se encuentra suscrita por los modos en que las organizaciones potencian, crean, generan, organizan y difunden nuevos conocimientos y rutinas de actividades, dentro de sus propios climas organizacionales. En este sentido, es necesario que gerencialmente se tomen decisiones adecuadas de inversión en tecnología, por cuanto contribuyen a mejorar su rentabilidad y posicionamiento competitivo, en la cual contribuye mucho contar con un personal calificado y actualizado para llevar a cabo tales actividades.

Asimismo, Boscán (2013) sostiene que el 80\% de los gerentes de las empresas de manufacturas plásticas entrevistados siempre (36,7\%), casi siempre $(33,3 \%)$ o algunas veces (10\%) facilita la interacción entre los trabajadores, para lograr la propagación de conocimientos técnicos, en tanto que el $20 \%$ casi nunca lo proporciona. Al respecto, el conocimiento contribuye, en sí mismo, a la ampliación de la base sobre la cual se sustenta su generación, de forma tal que posibilita el fortalecimiento de las propias capacidades de las empresas y las prepara en el marco de la resolución de sus problemas.

\section{Desarrollo urbano}

En la tabla 2, se indagó si el desarrollo urbano cerca de la empresa potencia su crecimiento, obteniéndose como resultado que el $46,7 \%$ de los gerentes de las empresas de manufacturas plásticas zulianas, considera que algunas veces el desarrollo urbano cerca de su empresa fortalece su crecimiento, el 20\% manifestó que casi siempre o siempre contribuye a su desarrollo, en tanto que el $33,4 \%$ sostuvo que casi nunca o nunca el desarrollo del territorio impulsa su crecimiento. Al respecto más del 60\% de los empresarios del sector, de acuerdo con Vázquez
(2007) aprovecha el territorio, como espacio de competitividad que induce a la empresa a responder estratégicamente a través de iniciativas locales que estimulan los procesos de desarrollo endógeno creando un entorno local favorable a la producción.

Tabla 2.

Desarrollo urbano como impulso al crecimiento.

\begin{tabular}{|l|c|c|}
\hline & Frecuencia & Porcentaje \\
\hline Nunca & 2 & 6,7 \\
\hline Casi Nunca & 8 & 26,7 \\
\hline Algunas Veces & 14 & 46,7 \\
\hline Casi Siempre & 5 & 16,7 \\
\hline Siempre & 1 & 3,3 \\
\hline Total & 30 & 100,0 \\
\hline
\end{tabular}

Fuente: Boscán (2013).

Cabe mencionar que actualmente las vías de acceso a la mayoría de las empresas del sector se encuentran en muy mal estado, sin embargo, la mayoría de los gerentes no han mostrado ningún interés en contribuir a la reparación de estas, considerando que es obligación del gobierno regional hacerlo, lo cual trae consecuencias para sus empresas, por cuanto dificulta la entrada y salida de estas, tanto de trabajadores como de potenciales clientes, frenando el desarrollo de esas empresas.

\section{Entorno institucional}

Aproximadamente el 80\% "(79,9\%) de los empresarios del sector de manufacturas plásticas, consideran que algunas veces $(53,3 \%)$, casi siempre $(23,3 \%)$ o siempre $(3,3 \%)$ el entorno institucional es favorable para impulsar el desarrollo endógeno de sus empresas, mientras que solo el $20 \%$ manifestó que casi nunca o nunca la densidad del tejido institucional contribuye 
a propiciar el desarrollo de sus organizaciones" (Boscán, 2013, p.198). En este sentido, la mayoría de los gerentes entrevistados estaría de acuerdo con los planteamientos de Vázquez (2007) quien sostiene que cada sociedad alienta el desarrollo de instituciones que le son propias y que facilitarán o dificultarán la actividad económica debido a que los agentes económicos toman sus decisiones en ese entorno organizativo e institucional y no siempre siguen las prescripciones teóricas de los modelos económicos.

Por otro lado, adicionalmente a los elementos antes mencionados y de acuerdo con Mas (2006), una magnitud abundante de mano de obra, sin capacidades para lograr una incorporación a los sistemas tecnológicos, así como a los procesos técnicos, no garantiza la eficiencia ni utilidades necesarias para el desenvolvimiento de una empresa. Por tanto, en la medida en que el personal está capacitado, permeado de conocimientos al igual que de avance profesional, puede generar efectos productivos que permiten incrementar la productividad de una empresa, así como desarrollar, a partir de la creación, perfeccionamiento de las habilidades para el trabajo, la capacidad de asumir los cambios para lograr mejoras tanto financieras como técnicas. Por lo cual a continuación se indaga acerca de las capacidades del personal en el sector de manufacturas plástica del estado Zulia.

\section{Capacidades del personal}

En el siguiente tabla 3, se puede observar que el $86,7 \%$ de los gerentes de las empresas de manufacturas plásticas, casi siempre, siempre o algunas veces impulsan la capacitación del personal, con el propósito de alcanzar el desarrollo endógeno de las mismas, y está capacitado para asumir los cambios tecnológicos que se lleven a cabo dentro de la empresa, en tanto que solo el 13,3\% reveló que casi nunca fortalece la formación de sus trabajadores. En este sentido, la capacitación del personal es fundamental para la incorporación de innovaciones tecnológicas en el proceso productivo, así como también facilita la introducción de nuevas maquinarias y equipos, nuevos métodos, la automatización de procesos, entre otros. Por lo tanto, contar con un personal capacitado, por un sistema de formación profesional construido por la localidad y por consolidación histórica de conocimientos (experiencias), muy notorio en este sector en los actuales momentos, provoca el incremento de la productividad del trabajo, generando un incremento del ingreso y favoreciendo los procesos de desarrollo en la empresa.

Tabla 3.

Impulso a la capacitación del personal

para el desarrollo endógeno

\begin{tabular}{|l|c|c|}
\hline & Frecuencia & Porcentaje \\
\hline Casi Nunca & 4 & 13,3 \\
\hline Algunas Veces & 5 & 16,7 \\
\hline Casi Siempre & 15 & 50,0 \\
\hline Siempre & 6 & 20,0 \\
\hline Total & 30 & 100,0 \\
\hline
\end{tabular}

Fuente: Boscán (2013).

\section{Tecnología}

De igual forma es importante examinar si la empresa cuenta con el nivel tecnológico apropiado para alcanzar el desarrollo, evidenciándose en la tabla 4, que el 76,6\% de los gerentes entrevistados, casi siempre, siempre o algunas veces, cuentan con el nivel tecnológico adecuado para impulsar el desarrollo endógeno, en tanto que el 23,3\% de los empresarios del sector de manufacturas plásticas zuliano, manifestó que casi nunca o nunca poseen un nivel tecnológico que favorezca el desarrollo en su empresa. Este resultado, es fundamental para el desarrollo de la esfera productiva o econó- 
mica, dado que los cambios en los procesos de creación, adaptación, innovación, transferencia y uso de las plataformas tecnológicas permiten realizar los cambios organizacionales.

En este sentido, según las últimas entrevistas realizadas a algunos empresarios del sector, existe una relación importante entre la tecnología y las capacidades del personal, pues son éstas últimas las que pueden crear y transformar conocimiento suficiente, generando a su vez, innovación que permite lograr que la tecnología se convierta en el factor estratégico de ganancia de la economía.

Tabla 4.

Nivel tecnológico apropiado para

alcanzar el desarrollo endógeno.

\begin{tabular}{|l|c|c|}
\hline & Frecuencia & Porcentaje \\
\hline Nunca & 1 & 3,3 \\
\hline Casi Nunca & 6 & 20,0 \\
\hline Algunas Veces & 4 & 13,3 \\
\hline Casi Siempre & 13 & 43,3 \\
\hline Siempre & 6 & 20,0 \\
\hline Total & 30 & 100,0 \\
\hline
\end{tabular}

Fuente: Boscán (2012).

De nada sirve contar con un nivel tecnológico adecuado para impulsar el desarrollo de la empresa, sino se les permite a los trabajadores el acceso a la tecnología y a los cambios que éstos pueden generar en ella. Al respecto según Boscán (2013), el 80\% de los gerentes de las empresas de manufacturas plásticas zulianas, facilitan el acceso de los trabajadores a nuevas tecnologías.

\section{Creación y adaptación de tecnología}

De acuerdo con Boscán (2013), el 66,6\% de los empresarios del sector de manufacturas plás- ticas, casi nunca o nunca desarrolla cambios tecnológicos en su empresa, mientras que $33,3 \%$ de los gerentes entrevistados sostuvieron que han realizado avances tecnológicos en los procesos de producción. En este sentido, cabe mencionar que los cambios tecnológicos, comprenden modificaciones en la forma como el trabajo se desempeña o los métodos y equipos utilizados, impulsando las innovaciones.

Por su parte, en el gráfico II se puede apreciar que el 76,7\% de los gerentes de las empresas de manufacturas plásticas zulianas, reveló que casi nunca o nunca favorece la creación propia de tecnología, lo cual, imposibilita el dinamismo y sostenibilidad del desarrollo económico de las empresas.

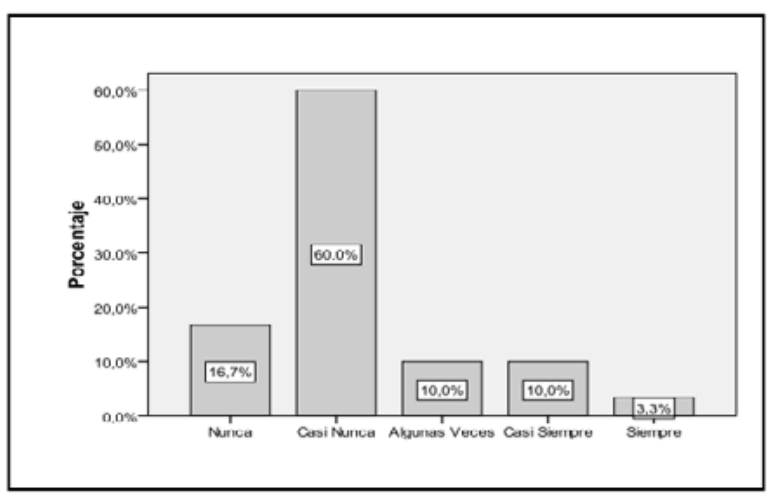

Gráfica 2: Generación propia de tecnología.

Fuente: Boscán (2013).

Por otro lado, Boscán (2013) sostiene que el $73,4 \%$ de los empresarvios del sector de manufacturas plásticas en el estado Zulia, manifestó que algunas veces (36,7\%), casi siempre $(26,7 \%)$ o siempre (10\%), que se ha adquirido alguna tecnología en la empresa han tenido que realizarles adaptaciones, mientras que el $26,6 \%$ de los gerentes, han considerado que casi nunca o nunca les han realizado ninguna adaptación. Al respecto, en la actualidad la mayoría de los empresarios ha estado de acuerdo en la adaptación tecnológica por cuanto estas son importadas y necesitan ser ajustadas o adecuadas a 
los requerimientos específicos de cada empresa, en concordancia con la estrategia económica para el desarrollo de esta. Asimismo, sostienen que los ajustes que le han realizado a las tecnologías que han implementado en sus empresas, ha contribuido a alcanzar un mayor desarrollo en las mismas.

Como ya se mencionó anteriormente, la tecnología es uno de los factores fundamental del desarrollo económico. En este sentido y tal como lo sostiene Mas (2006), en la tecnología se fusiona todo cuanto el ser humano acumula como conocimiento para poder obtener un propósito, refiriéndose este último al saber obtenido como resultado de la experiencia empírica, la investigación científica o las técnicas asociadas en la acción. Por lo tanto, todo conocimiento disponible nutre la posibilidad de la creación o innovación de tecnología y no existe tecnología si previamente no se diseña y construye el conjunto de materias adecuadas que posibilitan su existencia, es por ello que la tecnología y la innovación conforman elementos claves que potencian el buen desenvolvimiento del desarrollo.

Por último, dado que en América Latina, se han venido implementando diversas estrategias que buscan la incorporación de productores al entorno eco-eficiente, como parte de un cambio de actitud, y que el desarrollo de un país requiere cambios importantes en los patrones de producción y consumo, que implique reducciones del impacto ambiental, mejorando significativamente el bienestar humano y el equilibrio ecológico, en las entrevistas realizadas a algunos empresarios del sector se indagó acerca de este tema.

En este sentido, los plásticos creados a partir de derivados del petróleo, gas natural y carbón son persistentes en el medio ambiente, es decir, generan residuos en grandes canti- dades y su degradación es lenta, no son biodegradables, a diferencia de los obtenidos de insumos de origen vegetal. Por tanto, un tratamiento inadecuado de eliminación de los residuos de materiales plásticos (especialmente sólidos y líquidos), es una fuente significativa de contaminación ambiental, además sin una adecuada deposición final atentan contra la biodiversidad, puesto que, los desechos plásticos pueden causar la muerte de seres vivos. Al respecto, algunas empresas manifestaron que no se generaban desechos en sus procesos productivos, pues parte del material sobrante se reciclaba dentro de la misma empresa o se aprovechaba comercialmente a través de la reventa entre empresas del sector.

Asimismo, en la actualidad un nuevo tipo de plásticos biodegradables (bioplásticos), elaborados a partir de una variedad de plantas y otros recursos renovables de origen natural (especialmente maíz, celulosa y almidón), han comenzado a revolucionar la industria a nivel internacional. Sostiene Verdú (2013), que el desarrollo de una Industria, basada en materiales procedentes, total o parcialmente, de fuentes renovables, se desprende del hecho que en Venezuela, a pesar de la situación actual del sector manufacturero, es obvio identificar que estas fuentes son productos clásicos, derivados del sector agrícola, y en algunos casos de desechos agroindustriales, que la industria podría estar en capacidad de producir, ya que la tecnología es accesible y las condiciones agroecológicas favorecen a ciertos cultivos. Al respecto, las autoras en conversaciones previas con algunos gerentes del sector en el estado Zulia, recabaron información acerca de algunas experiencias de una o dos empresas que han elaborado bolsas plásticas biodegradables, utilizando materias a base de maíz o almidón importadas en asociación con algún cliente especifico. 


\section{Conclusiones}

El análisis y discusión de los resultados anteriores permiten concluir, que los elementos determinantes del desarrollo en las empresas del sector plástico son claves para mantener e incrementar su participación en el mercado. En este sentido, la mayoría de los gerentes de dichas empresas, estimulan la difusión de las innovaciones, motivan la participación de sus trabajadores en cursos de actualización de conocimientos técnicos, así como facilitan la interacción entre los trabajadores, para lograr la propagación de esos conocimientos, considerando que el mismo constituye en la actualidad un factor productivo de relevancia fundamental, pues es decisivo para la introducción de innovaciones, que ayudan al desarrollo de la empresa.

Asimismo, los empresarios del sector de manufacturas plásticas en su mayoría consideran que el desarrollo urbano cerca de su empresa fortalece su crecimiento, sin embargo, no contribuye a mejorar la infraestructura vial para impulsar el desarrollo endógeno de su empresa. Por otra parte, el entorno gubernamental se considera favorable, por cuanto se manifiesta que el Estado ofrece el soporte institucional, financiero e informativo, que se necesita para la adecuación del desarrollo endógeno en la localidad.

De igual manera, la mayoría de los empresarios del sector de manufacturas plásticas zuliano no desarrolla cambios tecnológicos en los procesos productivos de sus empresas y por ende no favorece la generación propia de tecnología. No obstante, la mayor parte de los gerentes entrevistados reveló que realizan adaptaciones a la tecnología que van a implementar en sus empresas y esos ajustes a su vez se traducen en un mayor desarrollo para éstas últimas.

Consecuentemente, el conjunto de estos facto- res o elementos fundacionales, presentes en las empresas de manufacturas plásticas zulianas, benefician el sostenimiento de condiciones a lo largo del tiempo, los cuales permiten a su vez, ejecutar los procesos de transformación para mantener, crear y evolucionar los soportes del desarrollo, con el propósito de alcanzar el éxito en el largo plazo. Por lo tanto, estas organizaciones requieren favorecer la generación propia de tecnología, utilizando los conocimientos adquiridos por sus trabajadores, empírica o científicamente, así como sus capacidades gerenciales y tecnológicas, considerando que el desarrollo endógeno viabilizado por la tecnología constituye una herramienta fundamental en la transformación y cambio social del país.

Finalmente, en el caso de las empresas del sector de manufactureras plásticas zuliano, salvo pocos casos particulares, ha sido difícil la utilización de materias primas biodegradables, así como la incorporación de tecnologías verdes, pues en general la actualización tecnológica constituye un problema para estas organizaciones. Por tanto, se plantea la necesidad de un esfuerzo conjunto entre la gerencia de estas organizaciones y los organismos de apoyo a la actividad productiva, para desarrollar programas de conversión tecnológica que permita al sector adecuarse a estándares internacionales y generar productos biodegradables. 


\section{Referencias}

Boscán, M. \& Sandrea, M. (2006). Estrategias de financiamiento para la incorporación de innovaciones tecnológicas impulsoras del desarrollo endógeno en el sector lácteo. Revista Estudios Iberoamericanos. 1(1). 9-28.

Boscán, M. (2013). Estrategias de financiamiento y desarrollo endógeno del sector plástico. (Tesis Doctoral Mención publicación). Universidad Dr. Rafael Belloso Chacín, Maracaibo, República Bolivariana de Venezuela.

Hernández, R., Fernández, C. \& Baptista, P. (2010). Metodología de la investigación. (5ta edición). México: McGraw-hill.

Hurtado, J. (2012). Metodología de la investigación. 4ta edición. Bogotá, Colombia: Ediciones Quiron - Sypal.

Mas Herrera, M. J. (2006). Desarrollo endógeno cooperación y competencia. Caracas, República Bolivariana de Venezuela: Editorial Panapo de Venezuela.

Ochoa, A. (2006). Aprendiendo en torno al Desarrollo Endógeno. Mérida, Venezuela: Editorial Fundacite Mérida.

Petrizzo Páez, M. A. (2008). El desarrollo endógeno y aprendizaje institucional: Una relación recursiva. Revista Venezolana de Gerencia.13 (41). 30-48.

Rodríguez, F. (2009). Una reflexión teórica sobre la innovación en el sector turismo. Revista Nicolaita de Estudios Económicos. 2 (4). 9-26.

Sandrea, M. \& Boscán, M. (2010). Gerencia ambiental en el sector zuliano de manufacturas plásticas. Revista espacio abierto. Cuaderno venezolano de sociología. 19(3). 555-571.

Vázquez Barquero, A. (2007). Desarrollo endógeno.
Teorías y políticas de desarrollo territorial Investigaciones regionales. Investigaciones Regionales. 11. 183-210.

Vázquez Barquero, A. (2018). Constitución, desarrollo endógeno y dinámica de las instituciones. Revista de Economía Mundial. 48. 201-220.

Velázquez Castro, J. A., Coria, E. C. \& Vargas Martínez, E. E. (2018). Cooperación empresarial para el fomento de la innovación en la pyme turística. Revista de Ciencias Sociales (RCS). 24 (3).

Verdú Arias, R. (2013). Los bioplásticos: un mundo de grandes posibilidades. Revista Conversaplast. 7(14). 24-32. 